\title{
COMBINATORIAL DIMENSION OF FRACTIONAL CARTESIAN PRODUCTS
}

\author{
RON C. BLEI AND JAMES H. SCHMERL
}

(Communicated by Jeffry N. Kahn)

\begin{abstract}
The combinatorial dimension of a fractional Cartesian product is the optimal value of an associated linear programming problem.
\end{abstract}

Fractional Cartesian products were introduced in a context of harmonic analysis to fill gaps between Cartesian products of spectral sets [1]. These fractional products subsequently gave rise to the idea of combinatorial dimension, a measurement of interdependencies that plays a natural role in harmonic and stochastic analysis (e.g., $[2,4,6])$. In this note, which is completely elementary and self-contained, we compute the combinatorial dimension of a general fractional Cartesian product by solving an associated linear programming problem.

Definition $1[2,5]$. Let $E_{1}, \ldots, E_{n}$ be infinite sets, and let $F \subset E_{1} \times \cdots \times E_{n}$. For an integer $s>0$, let

$$
\Psi_{F}(s)=\max \left\{\left|F \cap\left(A_{1} \times \cdots \times A_{n}\right)\right|: A_{i} \subset E_{i}, \quad\left|A_{i}\right| \leq s, \quad i=1, \ldots, n\right\} .
$$

Define the combinatorial dimension of $F$ to be

$$
\operatorname{dim} F=\limsup _{s \rightarrow \infty}\left(\ln \Psi_{F}(s) / \ln s\right) .
$$

Equivalently, for each real number $a>0$, let

$$
d_{F}(a)=\limsup _{s \rightarrow \infty}\left(\Psi_{F}(s) / s^{a}\right),
$$

and then $\operatorname{dim} F=\inf \left\{a: d_{F}(a)<\infty\right\}$.

The parameter $\operatorname{dim} F$, for $F \subset E_{1} \times \cdots \times E_{n}$, can be viewed as a measurement of interdependence between the canonical projections from $E_{1} \times \cdots \times E_{n}$ onto $E_{j}, j=1, \ldots, n$, restricted to $F$. The idea of combinatorial dimension was motivated at the outset by problems in harmonic analysis [1]. Indeed, it turns out that $\operatorname{dim} F$ can precisely be linked to harmonic-analytic parameters that are naturally associated with spectral sets $[2,3]$.

Clearly, $\operatorname{dim}\left(E_{1} \times \cdots \times E_{n}\right)=n$, and if $F \subset E_{1} \times \cdots \times E_{n}$ is infinite then $1 \leq \operatorname{dim} F \leq n$. For arbitrary $\alpha \in(1, n)$, randomly produced examples of $\alpha$-dimensional sets can be found in [5]. General fractional Cartesian products, archetypal instances of which appeared in [1,3], provide explicit examples

Received by the editors October 10, 1991 and, in revised form, May 4, 1992.

1991 Mathematics Subject Classification. Primary 05C65, 05C70; Secondary 43A46. 
of sets with fractional combinatorial dimension; we know of no other general scheme which produces explicit examples of sets with arbitrary fractional dimension.

For a positive integer $m$, let $[m]=\{1, \ldots, m\}$. Let $m$ be some fixed positive integer, and let $X$ be some fixed infinite set. For a subset $S \subset[\mathrm{m}]$, let $X^{s}$ be the set of all functions from $S$ into $X$. In particular, we can identify $X^{[m]}$ and $X^{m}$. If $S_{1} \subset S_{2} \subset[m]$, then we let $t \mapsto t \mid S_{1}$ be the projection from $X^{S_{2}}$ onto $X^{S_{1}}$; that is, if $t \in X^{S_{2}}$ then $t \mid S_{1} \in X^{S_{1}}$ is such that, for each $i \in S_{1}, \quad\left(t \mid S_{1}\right)(i)=t(i)$.

Definition 2. Let $X$ be an infinite set. Let $\mathscr{U}=\left\{S_{1}, \ldots, S_{n}\right\}$ be a cover of $[m]$; that is, $[m]=S_{1} \cup \cdots \cup S_{n}$. The fractional Cartesian product of $X$ based on $\mathscr{U}$ is

$$
X^{(\mathscr{U})}=\left\{\left(t\left|S_{1}, \ldots, t\right| S_{n}\right): t \in X^{m}\right\}
$$

Now let $\mathscr{U}=\left\{S_{1}, \ldots, S_{n}\right\}$ be a cover of $[m]$, and consider the problem of computing the combinatorial dimension of $X^{(\mathscr{U})}$, where $X^{(\mathscr{U})}$ is viewed as a subset of the $n$-fold Cartesian product $X^{S_{1}} \times \cdots \times X^{S_{n}}$. In the case that $\mathscr{U}$ consists of $k$-element subsets of $[m](k \geq 1)$ so that $\left|\left\{j: i \in S_{j}\right\}\right|=$ $I \geq 1$, for all $i \in[m]$, it is shown in [3, Corollary 2.6] that $\operatorname{dim} X^{(\mathscr{U})}=$ $m / k$. In the general case, we show that the combinatorial dimension of an arbitrary fractional Cartesian product is the optimal value of the following linear programming problem:

$$
\text { Maximize } x_{1}+\cdots+x_{m}
$$

$$
\text { subject to the constraints that each } x_{i} \geq 0 \text { and that }
$$

$$
\sum_{i \in S_{j}} x_{i} \leq 1 \text { for each } j \in[n]
$$

Since $\mathscr{U}$ is a cover of $[m]$, the feasible set is bounded, and thus the optimal value exists. Let $\alpha=\alpha(\mathscr{U})$ be this optimal value.

Theorem 3. $\operatorname{dim} X^{(U)}=\alpha(\mathscr{U})$.

Proof. First we prove the inequality $\alpha(\mathscr{U}) \leq \operatorname{dim} X^{(\mathscr{U})}$. Let $\left(x_{1}, \ldots, x_{m}\right)$ be an optimal vector; that is, $x_{1}, \ldots, x_{m}$ satisfy the constraints of the linear programming problem, and $\alpha=x_{1}+\cdots+x_{m}$. Moreover, by letting $\left(x_{1}, \ldots, x_{m}\right)$ be an extreme point of the feasible set, we can assume that each of $x_{1}, \ldots, x_{m}$ is rational. Let $s$ be an arbitrarily large integer such that $s^{x_{i}}$ is an integer for each $i \in[m]$. Let $D_{1}, \ldots, D_{m} \subset X$ be such that $\left|D_{i}\right|=s^{x_{i}}$ for each $i \in$ [m] , and consider $D=D_{1} \times \cdots \times D_{m} \subset X^{m}$. For each $j \in[n]$, let $A_{j}=$ $\times\left\{D_{i}: i \in S_{j}\right\}$. We have $A_{j} \subset X^{S_{j}}$ and obtain from the constraints in (5)

$$
\left|A_{j}\right|=\prod_{i \in S_{j}}\left|D_{i}\right|=\prod_{i \in S_{j}} s^{x_{i}} \leq s .
$$

Also, it is clear that $t \mapsto\left(t\left|S_{1}, \ldots, t\right| S_{n}\right)$ is a bijection from $D$ onto $X^{(\mathscr{U})} \cap$ $\left(A_{1} \times \cdots \times A_{n}\right)$, so that

$$
\left|X^{(\mathscr{U})} \cap\left(A_{1} \times \cdots \times A_{n}\right)\right|=|D|=\prod_{i=1}^{n} s^{x_{i}}=s^{\alpha} .
$$


Since $s$ is an arbitrary integer, (6) and (7) imply that $\alpha \leq \operatorname{dim} X^{(\mathscr{U})}$.

To prove $\alpha \geq \operatorname{dim} X^{(\mathscr{U})}$, we consider the following dual linear programming problem:

$$
\text { Minimize } y_{1}+\cdots+y_{n}
$$

subject to the constraints that each $y_{j} \geq 0$ and that

$$
\sum_{i \in S_{j}} y_{j} \geq 1 \text { for each } i \in[m] .
$$

By the fundamental duality theorem of linear programming, the value of the dual problem exists and equals $\alpha=\alpha(\mathscr{U})$. Let $\left(y_{1}, \ldots, y_{n}\right)$ be an optimal vector. Let $A_{1}, \ldots, A_{n}$ be arbitrary finite subsets of $X^{S_{1}}, \ldots, X^{S_{n}}$, respectively. We will show

$$
\left|X^{(\mathscr{U})} \cap\left(A_{1} \times \cdots \times A_{n}\right)\right| \leq\left|A_{1}\right|^{y_{1}} \cdots\left|A_{n}\right|^{y_{n}} .
$$

In the case $\left|A_{1}\right| \leq s, \ldots,\left|A_{n}\right| \leq s,(9)$ of course implies

$$
\Psi_{X^{(\mathscr{U})}}(s) \leq s^{\sum_{j} y_{j}}=s^{\alpha}
$$

establishing $\alpha \geq \operatorname{dim} X^{(\mathscr{U})}$.

We use the notation: if $S=\left\{i_{1}, \ldots, i_{k}\right\}$ then $\sum_{t_{i} \in X: i \in S} f\left(t_{1}, \ldots, t_{m}\right)$ denotes the iterated sum

$$
\sum_{t_{i_{1}} \in X} \cdots \sum_{t_{i_{k}} \in X} f\left(t_{1}, \ldots, t_{m}\right) .
$$

In particular, if $S=\varnothing$ then $\sum_{t_{i} \in X: i \in S} f\left(t_{1}, \ldots, t_{m}\right)=f\left(t_{1}, \ldots, t_{m}\right)$. Observe that

$$
\left|X^{(\mathscr{U})} \cap\left(A_{1} \times \cdots \times A_{n}\right)\right|=\sum_{t_{i} \in X: i \in[m]} \prod_{j=1}^{n} \mathbf{1}_{A_{j}}\left(t_{i}: i \in S_{j}\right)
$$

$\left(\mathbf{1}_{A}\right.$ denotes the indicator function of $A$ ). To obtain (9), we apply recursively in (11) the following multilinear Hölder inequality: Let $k>1$ be an integer and let $p_{1} \in(0, \infty], \ldots, p_{k} \in(0, \infty]$ be such that $\left(1 / p_{1}\right)+\cdots+\left(1 / p_{k}\right) \geq 1$. Let $f_{1}, \ldots, f_{k}$ be real-valued functions defined on $X$. Then

$$
\sum_{t \in X}\left|f_{1}(t) \cdots f_{k}(t)\right| \leq\left\|f_{1}\right\|_{p_{1}} \cdots\left\|f_{k}\right\|_{p_{k}}
$$

$\left(\|\cdot\|_{p}\right.$ denotes the usual $l^{p}$-norm). For $q=0, \ldots, m$, define

$$
F(q)=\sum_{t_{i} \in X: i \in[m] \sim[q]} \prod_{j=1}^{n}\left(\sum_{t_{i} \in X: i \in[q] \cap S_{j}} \mathbf{1}_{A_{j}}\left(t_{i}: i \in S_{j}\right)\right)^{y_{j}} .
$$

Observe that $F(0)=\left|X^{(\mathscr{U})} \cap\left(A_{1} \times \cdots \times A_{n}\right)\right|$ and that $F(m)=\left|A_{1}\right|^{y_{1}} \cdots\left|A_{n}\right|^{\nu_{n}}$, so (9) is just $F(0) \leq F(m)$. We let $q=0, \ldots, m-1$ and proceed to show $F(q) \leq F(q+1)$, thus establishing (9). First we rewrite $F(q)$ as

$$
\begin{aligned}
F(q)= & \sum_{t_{i} \in X: i \in[m] \sim[q+1]} \prod_{\left\{j: q+1 \notin S_{j}\right\}}\left(\sum_{t_{i} \in X: i \in[q] \cap S_{j}} \mathbf{1}_{A_{j}}\left(t_{i}: i \in S_{j}\right)\right)^{y_{j}} \\
& \cdot \sum_{t_{q+1} \in X} \prod_{\left\{j: q+1 \in S_{j}\right\}}\left(\sum_{t_{i} \in X: i \in[q] \cap S_{j}} \mathbf{1}_{A_{j}}\left(t_{i}: i \in S_{j}\right)\right)^{y_{j}} .
\end{aligned}
$$


Given the constraints in the dual linear programming problem (8) $\left(\sum_{q+1 \in S_{j}} y_{j} \geq\right.$ 1 ), we apply (12) in (14) to the summation over $t_{q+1}$ with exponents $1 / y_{j}$ and functions $\left(\sum_{t_{j} \in X: i \in[q] \cap S_{j}} \mathbf{1}_{A_{j}}\left(t_{i}: i \in S_{j}\right)\right)^{y_{j}}$, where $q+1 \in S_{j}$. We thus obtain

$$
\begin{aligned}
F(q) \leq & \sum_{t_{i} \in X: i \in[m] \sim[q+1]} \prod_{\left\{j: q+1 \notin S_{j}\right\}}\left(\sum_{t_{i} \in X: i \in[q] \cap S_{j}} \mathbf{1}_{A_{j}}\left(t_{i}: i \in S_{j}\right)\right)^{y_{j}} \\
& \cdot \prod_{\left\{j: q+1 \in S_{j}\right\}}\left(\sum_{t_{q+1} \in X} \sum_{t_{i} \in X: i \in[q] \cap S_{j}} \mathbf{1}_{A_{j}}\left(t_{i}: i \in S_{j}\right)\right)^{y_{j}} \\
= & \sum_{t_{i} \in X: i \in[m] \sim[q+1]} \prod_{j=1}^{n}\left(\sum_{t_{i}: i \in[q+1] \cap S_{j}} \mathbf{1}_{A_{j}}\left(t_{i}: i \in S_{j}\right)\right)^{y_{j}} \\
= & F(q+1) .
\end{aligned}
$$

Remarks. 1. Since the optimal value of the linear programming problem in (5) is a rational number, we deduce that, for every positive integer $m$ and every cover $\mathscr{U}$ of $[m]$, the combinatorial dimension of $X^{(\mathscr{U})}$ is rational.

2. $F \subset E_{1} \times \cdots \times E_{n}$ was said in [5] to be a $\gamma$-Cartesian product if

$$
0<\liminf _{s \rightarrow \infty}\left(\Psi_{F}(s) / s^{\gamma}\right) \leq \limsup _{s \rightarrow \infty}\left(\Psi_{F}(s) / s^{\gamma}\right)<\infty .
$$

Clearly, the combinatorial dimension of a $\gamma$-Cartesian product is $\gamma$. However, the statement in (16) is stronger than the statement $\operatorname{dim} F=\gamma$; this can be illustrated by the random constructions in [5]. In the framework of the present paper, following an elementary computation based on (6), (7), and (9), we observe that, for $\alpha=\alpha(\mathscr{U})$,

$$
\lim _{s \rightarrow \infty} \Psi_{X^{(\mathscr{Z})}}(s) / s^{\alpha}=1 .
$$

3. Corollary 2.6 in [3] can be directly deduced from our main theorem. Suppose that a cover $\mathscr{U}=\left\{S_{1}, \ldots, S_{n}\right\}$ of $[\mathrm{m}]$ consists of $k$-element subsets of $[m] \quad(k \geq 1)$ so that $\left|\left\{j: i \in S_{j}\right\}\right|=I \geq 1$ for all $i \in[m]$. Then, by a standard argument, the optimal value of the linear programming problem (5) is $\geq m / k$, and that of its dual (8) is $\leq n / I=m / k$. Therefore, by Theorem 3 and the fundamental duality theorem, $\operatorname{dim} X^{(\mathscr{Q})}=m / k$.

\section{REFERENCES}

1. R. C. Blei, Fractional Cartesian products of sets, Ann. Inst. Fourier (Grenoble) 29 (1979), 79-105.

2. __ Combinatorial dimension and certain norms in harmonic analysis, Amer. J. Math. 106 (1984), 847-887.

3. _ Fractional dimensions and bounded fractional forms, Mem. Amer. Math. Soc., vol. 57, Amer. Math. Soc., Providence, RI, 1985.

4. _ Stochastic integrators indexed by a multi-dimensional parameter, Probab. Theory Related Fields 95 (1993), 141-153. 
5. R. C. Blei and T. W. Körner, Combinatorial dimension and random sets, Israel J. Math. 47 (1984), 65-74.

6. R. C. Blei and J.-P. Kahane, A computation of the Littlewood exponent of stochastic processes, Math. Proc. Cambridge Philos. Soc. 103 (1988), 367-370.

Department of Mathematics, University of Connecticut, Storrs, Connecticut 06269 E-mail address, R. C. Blei: blei@uconnvm.uconn.edu

E-mail address, J. H. Schmerl: schmerl@uconnvm.uconn.edu 